\title{
Longitudinal Flow of Curviline Rotation Deflector by Viscous Incompressible Liquid
}

\author{
${ }^{1}$ Alexander I. Esin, ${ }^{\text {2 }}$ Dmitry A. Soloviev, ${ }^{3}$ Dmitry A. Kolganov, ${ }^{4}$ Dmitry G. Goryunov, ${ }^{5}$ Andrey V. \\ Kirichenko \\ 1,2,3,4,5 Saratov State Agrarian University named after N.I. Vavilov, Russia, Saratov \\ Email: esinai@yandex.ru,solovevda@bk.ru,dmi.kolg@mail.ru,md111@bk.ru,andreikirichenko@yandex.ru
}

Received: 16th January 2020, Accepted: 31st January 2020, Published: 30th April 2020

\begin{abstract}
The article considers longitudinal axisymmetric flow of curved rotation deflector with an averaged turbulent flow of incompressible fluid. Based on the Bernoulli equation, they determined the thickness of the water film descending from the deflector. They proved that the film thickness depends on the hydraulic resistance of the deflector. The jet departure range (deflector radius) was determined.
\end{abstract}

\section{Keywords}

Turbulent Motion, Curved Rotation Deflector, The Thickness of the Water Film, Bernoulli Equation, Generalized Solution, Correction Factor, Consideration of Friction Forces, Jet Departure Range.

\section{Introduction}

The flow of deflectors is of great practical importance for short-jet sprinkler nozzle design. The fluid flow flowing around the deflector takes on a fairly stable film shape, which remains at a certain distance from the vanishing point of the deflector surface, after which it loses stability, breaking up into separate drops.

\section{Methodology}

The motion of a turbulent liquid jet in air is so complex that it is not currently possible to describe this process on the basis of the Navier - Stokes differential equation solutions [1]. According to the experiments [2, 3], the decay of the jet begins from the peripheral part; therefore, the drops that are formed in the central zone of the jet have the greatest range. The point of their fall is the point of jet maximum departure. In this case the term "jet path" refers to any axial section of an axisymmetric flow. At that they assume that the transverse dimensions of the compact part of the liquid film are small as compared to the range and height of departure. In fact, it is believed that the entire liquid which moves along one path connecting the center of the living section of the film and the point of maximum departure, is the envelope of all jet drop paths [2]. The transverse dimensions of the compact part of the liquid film in the axisymmetric case are determined by the thickness of the film coming down from the deflector. Historically, the determination of the film thickness, as well as the force interaction between the liquid and the deflector, is calculated without taking into account the friction forces [2 - 6].

\section{Result \& Discussion}

Next, they consider the steady-state longitudinal flow of averaged turbulent fluid from a curvilinear rotation deflector with a characteristic angle $\beta$ (Fig. 1).

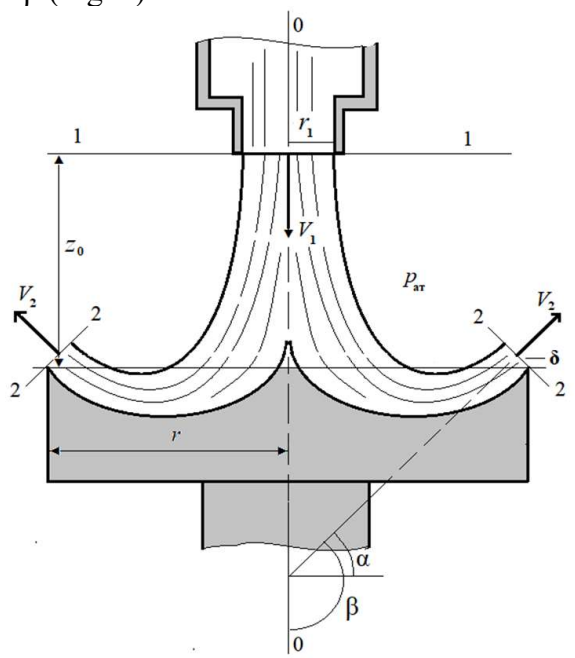

Fig. 1: Deflector Flow Pattern 
The distance $Z_{0}$ is assumed to be at least $r_{1}$, while the deflector does not affect the nature of the outflow [3]. Assuming the motion in the calculated sections changes smoothly, and neglecting the height $Z_{0}$ in comparison with the velocity heads, we will compose the Bernoulli equation for the sections $1-1,2-2$ :

$$
\alpha_{1} \frac{V_{1}^{2}}{2 g}=\alpha_{2} \frac{V_{2}^{2}}{2 g}+h_{f}
$$

where $h_{f}$ is the total pressure loss determined by the work of the friction forces on the surface of the deflector and on the liquid-air interface; $V_{1}, V_{2}$ are the average fluid velocities in the sections $1-1$ with the area $\omega_{1}=\pi r_{1}{ }^{2}$ and $2-2$ with the area $\omega_{2}=\pi \delta\left(2_{r}+\delta \cos \beta\right)$, respectively; $\delta$ is the film thickness in the section $2-2 ; \alpha_{1}, \alpha_{2}-$ Coriolis coefficients.

Having expressed the total pressure loss according to the Weisbach formula [7] $h_{f}=\zeta \frac{V_{2}^{2}}{2 g}$, we obtain the following:

$$
V_{1}=k V_{2}
$$

where

$$
k=\sqrt{\frac{\alpha_{2}+\zeta}{\alpha_{1}}}
$$

- the correction factor taking into account the work of friction forces.

Eliminating the average velocities through the flow rate $Q$ and the living cross-sectional areas $\omega_{1}, \omega_{2}$ from the equation (2), we will put it down in the following form:

$$
\omega_{2}=k \omega_{1}
$$

Substituting the areas of live sections $\omega_{1}, \omega_{2}$ in (4), we find the equation for film thickness determination like in [8]:

$$
\delta^{2}-\frac{2 r}{\sin \alpha} \delta+k \frac{r_{1}^{2}}{\sin \alpha}=0
$$

the solution of the quadratic equation (5) has the following form:

$$
\delta=\frac{r}{\sin \alpha} \pm \sqrt{\frac{r^{2}}{\sin ^{2} \alpha}-k \frac{r_{1}^{2}}{\sin \alpha}} .
$$

The sign in the formula (6) is selected from the following considerations:

- if $\alpha>0\left(\beta>\frac{\pi}{2}\right)$, the sign "._" is taken, otherwise the film thickness will be comparable with $\frac{r}{\sin \alpha}$, which contradicts the experiments;

- if $\alpha<0\left(\beta<\frac{\pi}{2}\right)$, the sign "+" is taken [8].

Then, let's limit ourselves to the consideration of the first case:

$$
\delta=\frac{r}{\sin \alpha}-\sqrt{\frac{r^{2}}{\sin ^{2} \alpha}-k \frac{r_{1}^{2}}{\sin \alpha}} .
$$

It follows from (7) that the film thickness increases with resistance coefficient $\zeta$ increase, and at $\alpha=0^{\circ}$ from (5) we obtain the following:

$$
\delta=0,5 k \frac{r_{1}^{2}}{r} .
$$

The formulas $(7,8)$ summarize the results $[2,3]$ if the work of friction forces is taken into account. The formula (7) can be converted to the following dimensionless form: 


$$
\frac{\delta}{r} \sin \alpha=1-\sqrt{1-k\left(\frac{r_{1}}{r} \sqrt{\sin \alpha}\right)^{2}} .
$$

The graph of dependence (9) for various values of the parameter k is shown in Fig. 2.

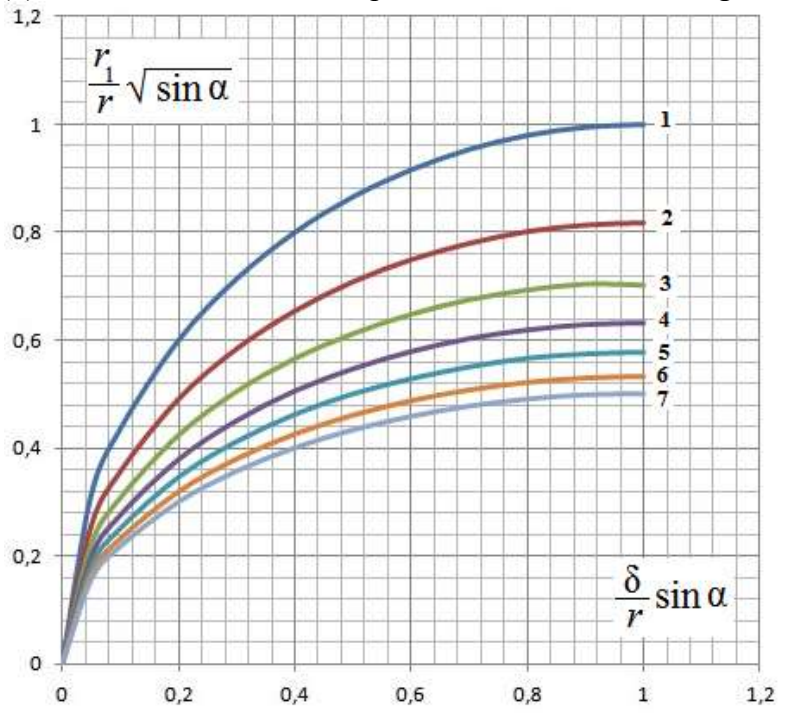

Fig. 2: Graphical Representation of the Dependence (9)

$$
\begin{gathered}
1-\mathrm{k}=1 ; 2-\mathrm{k}=1,5 ; 3-\mathrm{k}=2,0 ; 4-\mathrm{k}=2,5 \\
5-\mathrm{k}=3,0 ; 6-\mathrm{k}=3,5 ; 7-\mathrm{k}=4,0
\end{gathered}
$$

According to [5], the force of the jet $\mathrm{P}$ dynamic action on the deflector can be determined by the following formula:

$$
P=\rho V_{1} \omega_{1}\left(V_{1}-V_{2} \cos \beta\right),
$$

where $\rho$ is the fluid density. Expressing the average speed $V_{1}, V_{2}$ from the equation (2), we obtain the following:

$$
P=\rho V_{1}^{2} \omega_{1}\left(1+\frac{\sin \alpha}{k}\right)
$$

which is the generalization of the well-known formula for the force of jet dynamic action on a solid surface [5, 9].

In contrast to [7], where energy losses due to curvature of streamlines are taken into account by epy empirical factor equal to $0,92 \ldots 0,95$, in formula (10) all energy losses are taken into account by the coefficient $k$. From the equation (5), we can conclude that the coefficient $\mathrm{k}$ can be determined empirically, provided that the dimensions of the deflector, the film thickness, and the film exit angle $\alpha$ are known (see Fig. 1).

Neglecting the wind speed, the trajectory and length of the jet (the nozzle radius) $L$ will be presented in accordance with the diagram on Fig. 3, where the origin is placed at the gravity center of the living section $2-2$ (see Fig. 1), $Z_{n o z}+0,5 \delta \cos \alpha$-the distance from the gravity center of the living section $2-2$ to the earth surface.

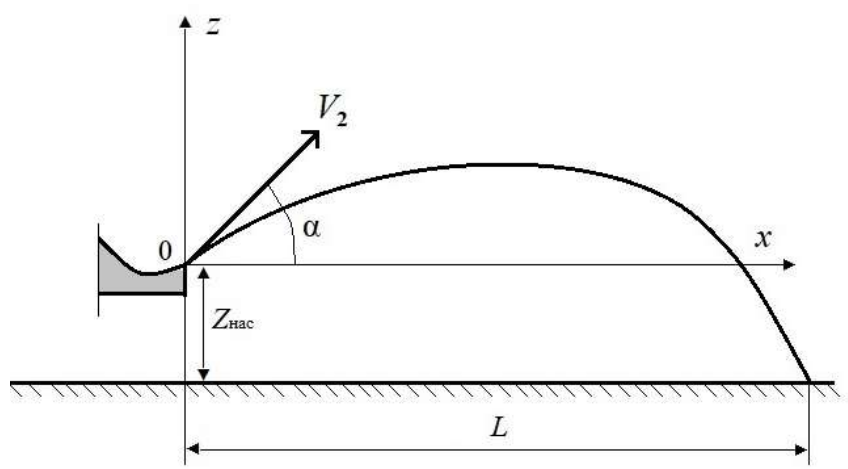

Fig. 3: The Scheme of the Jet Path Motion if there is No Wind 
Obviously $Z_{n o z} \gg 0,5 \delta \cos \alpha$, therefore we will take into account only $Z_{n o z}$ - the height of the deflector.

$$
z=x \operatorname{tg} \alpha-\frac{g}{2 V_{2}^{2} \cos ^{2} \alpha} x^{2} .
$$

Since the deflector fluid motion is axisymmetric, then the equation of the jet path can be written in the form [7] for any axial flow of the cross section in the absence of air resistance.

Excluding the speed $V_{2}$ using the equation (2), we obtain the following:

$$
z=x \operatorname{tg} \alpha-k^{2} \frac{g}{2 V_{1}^{2} \cos ^{2} \alpha} x^{2} .
$$

The speed $V_{1}$ is expressed by the formula for the cylindrical nozzle [7]

$$
V_{1}=\mu \sqrt{2 g H}
$$

where $\mu$ - the coefficient of nozzle speed (flow); $\mathrm{H}$ - the pressure in front of the nozzle.

Then the equation of the jet trajectory takes the following form:

$$
z=x \operatorname{tg} \alpha-\frac{k^{2}}{4 \mu^{2} H \cos ^{2} \alpha} x^{2} .
$$

At $\alpha<\frac{\pi}{4}$ the energy loss to overcome the air resistance can be approximately considered equal [7].

Where $\lambda$ - the resistance coefficient; $R_{2}-$ the hydraulic radius of the section $2-2$. Then the final form of the jet

$$
h_{f} \approx \lambda \frac{l}{4 R_{2}} \frac{V_{2}^{2}}{2 g}=\frac{\lambda}{k^{2}} \frac{x}{4 R_{2} \cos \alpha} \frac{\mu^{2} 2 g H}{2 g}=\frac{\lambda \mu^{2}}{4 k^{2}} \frac{H x}{R_{2} \cos \alpha},
$$

path is written as follows:

$$
z=x \operatorname{tg} \alpha-\frac{k^{2} x^{2}}{4 \mu^{2} H\left(1-\frac{\lambda \mu^{2}}{4 k^{2} R_{2} \cos \alpha} x\right) \cos ^{2} \alpha} .
$$

From the equation (11) one can find the equation to determine the radius $\mathrm{L}$ of the nozzle

$$
-z_{\text {нас }}=L \operatorname{tg} \alpha-\frac{k^{2} L^{2}}{4 \mu^{2} H\left(1-\frac{k^{2}}{4 \mu^{2}} \frac{K}{H \cos \alpha} L\right) \cos ^{2} \alpha},
$$

where

$$
K=\frac{\lambda \mu^{4}}{k^{4}} \frac{H}{R_{2}}
$$

is the dimensionless parameter depending, in the general case, on $r, r_{1}, \alpha, \delta, H$ and deflector and air resistance. To calculate the parameter $\mathrm{K}$, let's find the hydraulic radius $R_{2}$ of the living section $2-2$, which is a truncated cone with the generator $\delta$ (see Fig. 1)

$$
R_{2}=\frac{\omega_{2}}{\chi_{2}}=\frac{\pi \delta(2 r-\delta \sin \alpha)}{2 \pi r}=\delta-\frac{\delta^{2}}{2 r} \sin \alpha .
$$


Since $\left(\frac{\delta^{2}}{2 r}\right) \sin \alpha \ll \delta$, thus, following can be accepted

$$
\begin{gathered}
R_{2} \approx \delta . \\
K=\lambda \frac{\mu^{4}}{k^{4}} \frac{H}{\delta} .
\end{gathered}
$$

The friction coefficient $\lambda$ included in parameter (14) is generally determined by the Froude and Reynolds numbers, and according to A.I. Didebulidze's experiments [2], the friction coefficient $\lambda$ can be taken equal to $\lambda \approx$ $0,0018 \ldots 0,0020$ at Reynolds numbers of the order of $10^{5}$.

We will put down the quadratic equation (12) solution in the following form:

$$
\begin{gathered}
L=L_{0} \cos \alpha, \\
L_{0}=\frac{\sqrt{\left(a z_{\text {нас }} K-\sin \alpha\right)^{2}+4 a z_{\text {нас }}(1+K \sin \alpha)}-a z_{\text {нас }} K+\sin \alpha}{2 a(1+K \sin \alpha)}, \\
a=\frac{k^{2}}{4 \mu^{2} H} .
\end{gathered}
$$

To determine the maximum value of the nozzle action radius $L_{\text {max }}$, it is necessary to find the derivative $\frac{d_{L}}{d_{\alpha}}$ and equate it to zero. Since the derivative $\frac{d_{L}}{d_{\alpha}}$ has an extremely cumbersome form, it is simpler to organize the search for the quantity $L_{\max }$ numerically [10] according to the algorithm (14) - (17) without direct calculation of the derivative $\frac{d_{L}}{d_{\alpha}}$. Fig. 4 shows the graph of dependence (12) according to [12 - 16]

$$
Q=0,64 \frac{l}{\mathrm{~s}} ; d_{1}=10 \mathrm{~mm} ; d=50 \mathrm{~mm} ; \mu=0,82 ; Z_{\text {noz }}=0,5 \mathrm{~m},
$$

which shows that the maximum length of the jet departure (nozzle radius) $L_{\max }=1,5 \mathrm{~m}$ is achieved at $\alpha \approx$ $17^{\circ} \ldots 18^{\circ}$.

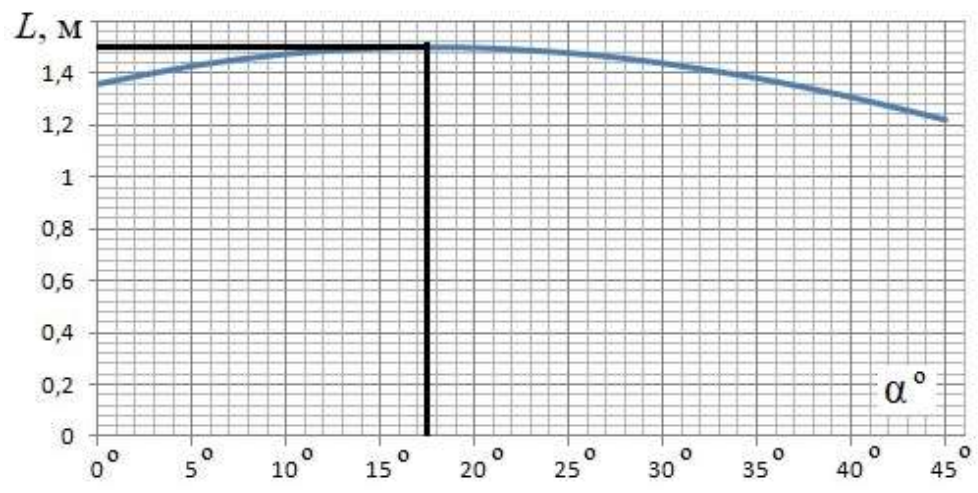

Fig. 4: Nozzle Radius Determination

If one neglects $Z_{n o z}$ in the equation (12) as compared with other terms of the equation (this is possible at $\alpha>$ $20^{\circ}$ ), then the radius $\mathrm{L}$ can be written as follows:

$$
L=\frac{2 \mu^{2} H \sin 2 \alpha}{k^{2}(1+K \sin \alpha)}
$$

which is the generalization of the known dependence [7]. 
Finding the derivative $\frac{d_{L}}{d_{\alpha}}$ and equating it to zero, we obtain the following:

$$
K \sin ^{3} \alpha+2 \sin ^{2} \alpha-1=-\frac{d K}{d \alpha} \sin ^{2} \alpha \cos \alpha .
$$

Any solution $\alpha$ of the equation (19) with a known value of the parameter $\mathrm{K}$ gives the maximum value of the nozzle radius. The derivative $\frac{d_{K}}{d_{\alpha}}$ entering into the equation (19) has the following form:

$$
\frac{d K}{d \alpha}=-\frac{K}{\delta} \frac{d \delta}{d \alpha}=-\frac{K}{2 \delta} \frac{2 r \delta-k r_{1}^{2}}{r-\delta \sin \alpha} \operatorname{ctg} \alpha .
$$

Taking into account the derivative (20), the equation (19) turns out to be the 5 th degree algebraic equation with respect to $\sin \alpha$ and it is unsolvable in radicals [11], therefore its solution can be found either numerically or graphically. According to (18), the graphical solution of the equation (19) is shown on Fig. 5.

On the other hand, calculations based on the dependence (14) show that the parameter K depends on the angle $\alpha$ very weakly (Fig. 6). When the angle $\alpha$ changes from $20^{\circ}$ to $45^{\circ}$ with the data (18), the value of K decreases by less than $0,5 \%$.

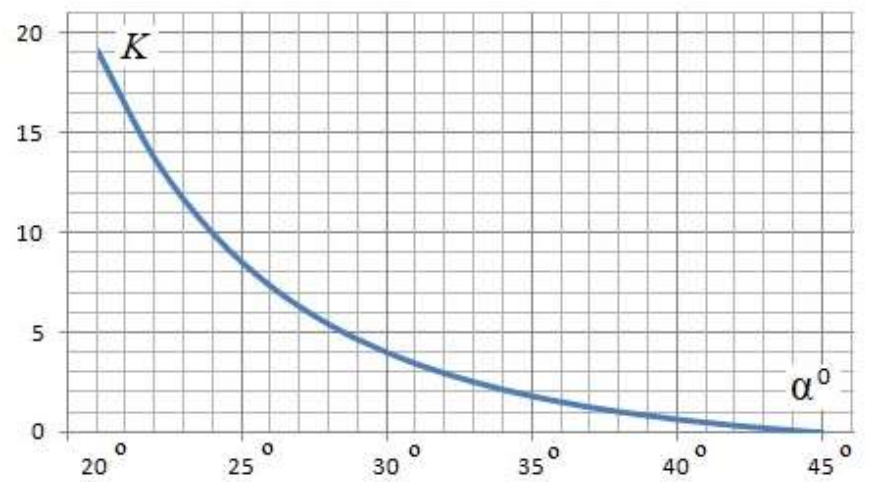

Fig. 5: Graphical Determination of the Jet Departure Optimum Angle

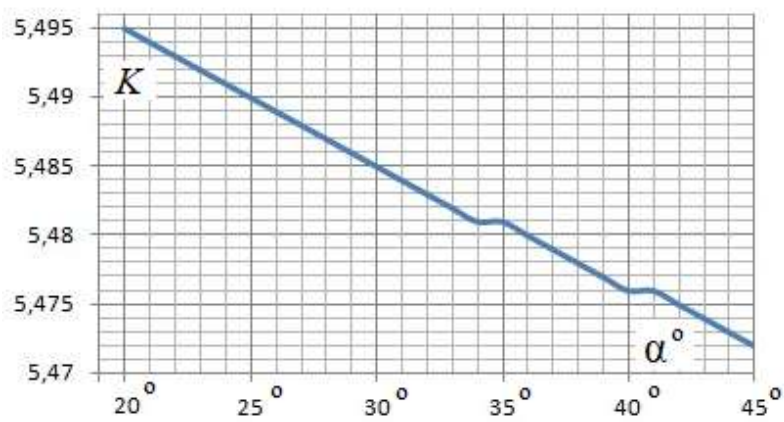

Fig. 6: The Dependence of the Parameter K on the Angle $\alpha$

Therefore, for approximate calculations, one can assume that $\mathrm{K}$ is independent of $\alpha\left(\frac{d_{K}}{d_{\alpha}}=0\right)$, then the equation (19) is simplified significantly, formally coinciding with [7]

$$
K \sin ^{3} \alpha+2 \sin ^{2} \alpha-1=0 .
$$

The solution of the equation (21) can be represented both graphically and analytically by the Cardano formulas [11]. 


\section{Conclusion}

The calculation of hydraulic jet trajectories according to equation (11) with the data (18) is shown on Fig. 7.

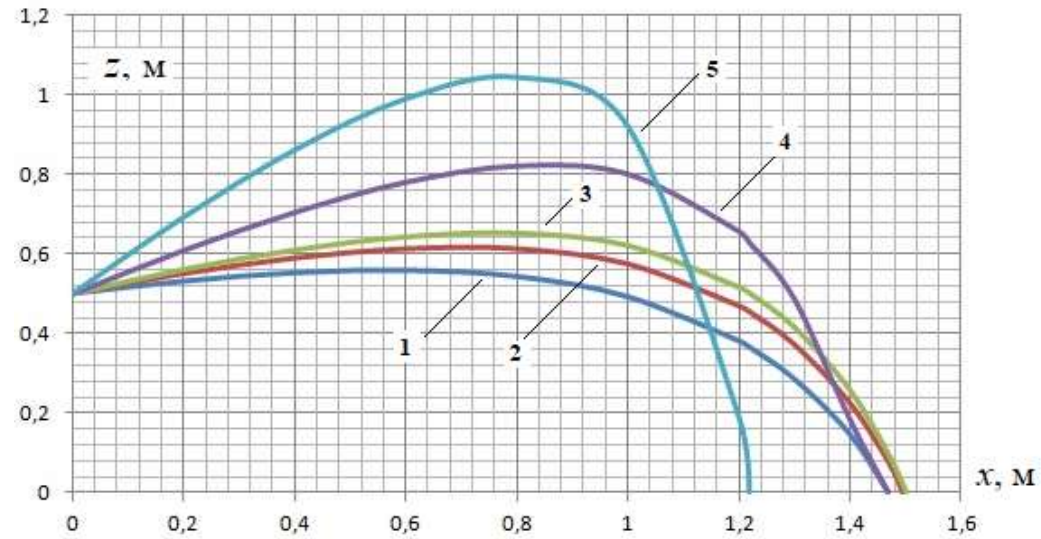

Fig. 7: The Trajectories of the Hydraulic Jets at Different Values of the Jet Departure Angle: $1-\alpha=10^{\circ}$; $2-\alpha=15^{\circ} ; 3-\alpha=18^{\circ} ; 4-\alpha=30^{\circ} ; 5-\alpha=4^{\circ}$

The obtained calculated dependences and the values of the given parameters were used to construct curved deflector nozzles, to determine the distance between the drainage systems of sprinklers and the sprinkler diameters for the sprinkler KASKAD [12-16] (Fig. 8, 9).

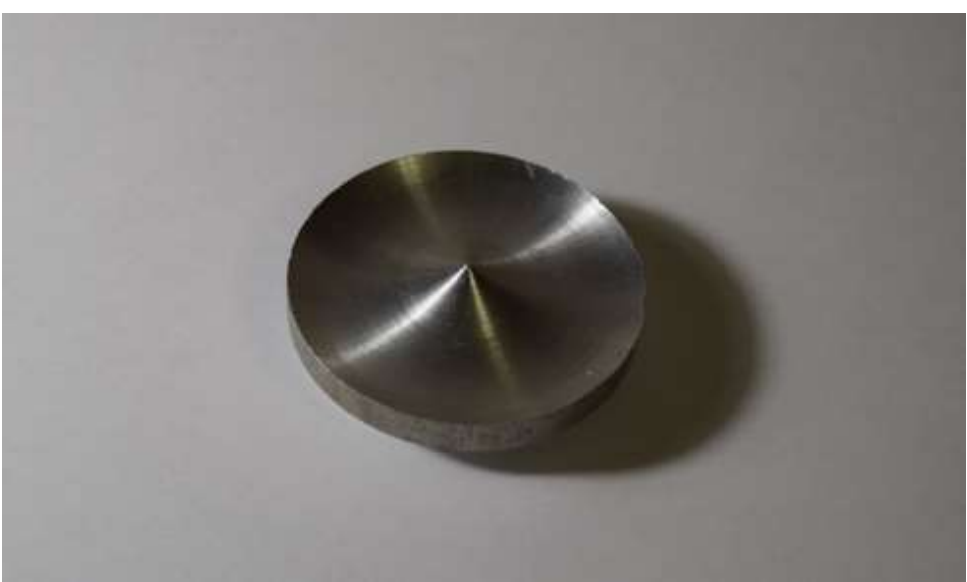

Fig. 8: One of the Models for Irrigation Nozzle

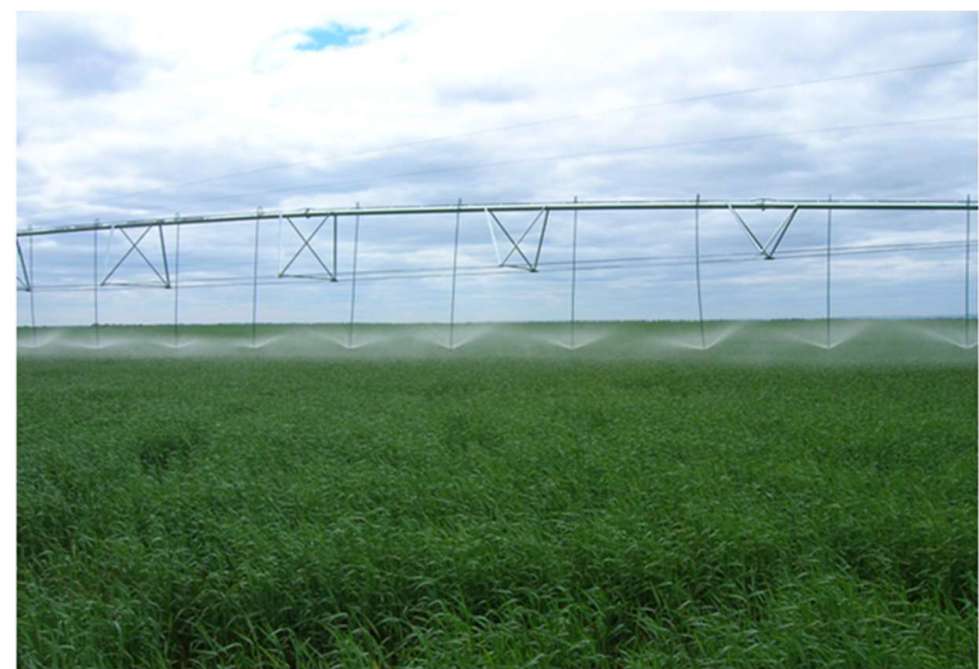

Fig. 9: The Sprinkler KASKAD 
The industrial production of the KASKAD sprinkler model range with a diverse range of components and additional options was launched in 2016. The machine was tested at the FSBI "Volga Region MIS", Samara. The Samara Center for Testing and Certification issued the Customs Union Certificate of Conformity "On machinery and equipment safety" (July 19, 2016).

\section{References}

1. Loitsyansky L.G. The mechanics of fluid and gas / L.G. Loitsyansky. - M.: Drofa, 2003. - 840 p.

2. Isaev, A.P. Hydraulics of sprinkling machines / A.P. Isaev. - M.: "Mechanical Engineering", 1973. - 216 p.

3. Lebedev, B.M. Sprinklers: theory and design / B.M. Lebedev. - M.: Mechanical Engineering, 1977. - 246 p.

4. Borodin, V.A. Liquid spraying / V.A. Borodin. - M.: Mechanical Engineering, 1967. - 263 p.

5. Bashta T.M. Hydraulics, hydraulic machines and hydraulic drives / T.M. Bashta et al. - M.: "Mechanical Engineering", 1982. - 423 p.

6. Pilgunov V.N. Verification of mathematical models for typical local hydraulic resistances [Electronic resource] / V.N. Pilgunov, K.D. Efremova // Engineering Herald. - 2013. - No. 11. - pp. 29 - 56. - Access mode: http://engsi.ru/issue/622075.html.

7. Shterenlikht D.V. Hydraulics / D.V. Sterenlicht. - M.: KolosS, 2004. - 656 p.

8. Yesin A.I. The flow of a conical deflector by viscous incompressible fluid. / A.I. Yesin, A.V. Rusinov, A.P. Akpasov, Yu.V. Bondarenko, L.A. Zhuravleva // Scientific life. - 2018. - No. 4. pp. 14 - 19.

9. Collection of tasks in engineering hydraulics: a manual for engineering universities / D.A. Butaev, Z.A. Kalmykova, L.G. Podviz and others; edited by I.I. Kukolevsky and L.G. Podviz. - M.: Mechanical Engineering, 1981. - 464 p.

10. Yesin A.I. Numerical hydraulics / A.I. Yesin. - Saratov: The Publishing house of Sarat. Agrarian University, 2013. - 160 p.

11. Korn G. Handbook of mathematics for scientists and engineers / G. Korn, T. Korn. - M.: Nauka, 1973. - 832 p.

12. Yesin A.I. Mathematical modeling of a water-conducting belt for sprinklers / A.I. Yesin, D.A. Soloviev, L.A. Zhuravleva // Scientific life. - 2017. - No. 9. pp. 20 -28.

13. Yesin A.I. The studies of water flow characteristics in the water supply belt of a sprinkler / A.I. Yesin, D.A. Soloviev, L.A. Zhuravleva // Scientific life. - 2018. - No. 2. pp. 16 - 25.

14. Yesin A.I. Recommendations for the selection of the sprinklers "KASKAD". / A.I. Yesin, D.A. Soloviev, L.A. Zhuravleva // Land reclamation and water management. 2018. No. 2. pp. 16-22.

15. Salnikov A.L. The results of KASKAD sprinkler experimental studies. / A.L. Salnikov, L.A. Zhuravleva, E.V. Smirnov, V.A. Soloviev // Scientific life. - 2018. - No. 7. pp. 31 - 37.

16. Esin A.I. Mathematical Modeling of water Conducting Belt for Circular Action Sprinkler / A.I. Esin, V.M. Boikov, V.A. Mukhin, F.V. Serebrennikov, L.A. Zhuravleva // Jour of Adv Research in Dynamical \& Control Systems, Vol. 10, 01-Special Issue, 2018. pp. $2135-2141$. 